- Questionnaires were sent to 8,355 women and 4,500 (55\%) were completed

- $88 \%$ of survey respondents were currently practising dentistry, mainly in general practice.

- Almost half of women dentists work full-time and half work part-time - regular hours. The main reason for choosing to work part-time was caring for children.

- 326 survey respondents were not currently practising; $60 \%$ were on maternity leave or caring for children, $20 \%$ had made a conscious decision not to continue to work as a dentist.

- Only 4\% of survey respondents had taken a career break and enrolled on KITS or GBTP courses.

- The results are important for women dentists, but are relevant to men too.

- This is the latest in a series of surveys which started 25 years ago.

\title{
Better opportunities for women dentists: a review of the contribution of women dentists to the workforce
}

\author{
J. J. Murray ${ }^{1}$ CBE
}

\begin{abstract}
In June 2000 the Department of Health commissioned a review to examine the need for improvements to the employment opportunities for women dentists in the National Health Service (NHS) across England. Dame Margaret Seward carried out the review, which was published in September 2001. ' The review was considered necessary for four main reasons. Firstly. workforce planning, because now more than 50\% of new entrants to dental undergraduate courses in the UK are female and by 2020 over $50 \%$ of all practising dentists will be female. Secondly, evidence that 50\% of women in dentistry work for no more than two days per week for the NHS. Thirdly, most women work either as associates in general dental practice (GDP) or in the Community Dental Service (CDS). Lastly, the perception that women find it difficult to return to dentistry after taking a career break.
\end{abstract}

\section{INTRODUCTION}

The report is divided into nine chapters and extends to 161 pages. This article is a personal précis of the review of the contribution of women dentists to the workforce. It summarises the quantitative and qualitative data that was collected so as to investigate specific areas of concern. These include:

- Experiences of women dentists in the General Dental Services (GDS).

- Reasons behind the decision to work part-time.

- Attractiveness of the Community Dental Service (CDS).

- Opportunities provided by the Personal Dental Service (PDS).

- Challenges women face in returning to dentistry after career breaks.

- Usefulness of the Keeping in Touch Scheme (KITS) and Getting Back to Practice (GBTP) courses.

- Other relevant issues.

$1^{*}$ Dean of Dentistry, Dental School, Framlington Place,

Newcastle upon Tyne, NE2 4BW

${ }^{*}$ Correspondence to: J. J. Murray

E-mail:j.murray@ncl.ac.uk

\section{Refereed Paper}

Received 9.11.01; Accepted 15.11.01

(๑) British Dental Journal 2002; 192: 191-196

\section{METHOD}

The study was divided into three parts:

- Self-completion questionnaire to all women dentists under 70 years of age on the General Dental Council Register, with a post code in England. (The questionnaire was also sent to those women dentists not currently registered whose names were obtained through a search process that included intensive coverage in the dental press.)

- Eight focus groups of women and men held in different parts of England.

- Written submissions requested from 54 leading organisations.

\section{RESULTS - QUESTIONNAIRE}

\section{Place of work}

Questionnaires were sent to 8,355 women and 4,500 (55\%) were completed. Of the 4,500 survey respondents 3,971 (88\%) reported that they were currently practising dentistry, mainly in general practice (Table 1). Less than one-third of those women currently practising were working as a practice owner, the remaining two-thirds were employed as an associate, an assistant or a vocational dental practitioner.

\section{Working patterns}

The working patterns of women dentists currently practising are given in Table 2 . Almost half reported that they were working full time. Most of those working parttime worked regular hours. The percentage of women working part-time, term time only was very small. Of those currently practising, 43\% reported that they were the main income provider in their household. The previous work patterns of the 529 dentists who were not currently working were different - only one-third had been working full-time and two-thirds had been working part-time.

For those currently practising parttime, the main reason was caring for children (65\%), but this was only narrowly ahead of personal choice (57\%). In fact, many respondents had indicated both reasons. Ill health accounted for 4\% working part-time, and caring for other relations 3\%. Of all the women responding, 49\% had tried to find part-time work, 92\% were successful (Table 3).

Potential changes that would attract respondents to work more hours in the NHS are highlighted in Figure 1. Lightening the administrative burden, changes in emergency cover requirements, availability of 


\section{Table 1 Field of dentistry for survey respondents by whether currently practising}

\begin{tabular}{|c|c|c|}
\hline Current or last field* & Currently practising (\%) & Not currently practising $(\%)$ \\
\hline General practice & 76 & 60 \\
\hline Corporate body & 4 & 2 \\
\hline Industrial & 1 & 1 \\
\hline Defence dental service & 1 & 1 \\
\hline Community dental service & 16 & 32 \\
\hline Hospital dental service & 11 & 10 \\
\hline University & 5 & 5 \\
\hline Personal dental service & 2 & 1 \\
\hline Health authority & 2 & 4 \\
\hline Other dental field & 3 & 3 \\
\hline Base: number of survey respondents (=100\%) & 3,971 & 529 \\
\hline
\end{tabular}

\section{Table 2 Working patterns for survey respondents currently practising}

\begin{tabular}{lr}
\hline & Currently practising $(\%)$ \\
\hline Full-time & 48 \\
Part-time: regular hours & 41 \\
Part-time: extremely flexible & 1 \\
Part-time: evenings & 0 \\
Part-time: term time only & 0 \\
Part-time combination & 5 \\
Full-time, part-time and other combination & 2 \\
Other & 2 \\
No answer & 1 \\
\hline Base: number of survey respondents (=100\%) & 3,971 \\
\hline
\end{tabular}

a wider range of materials and techniques were amongst the commonest potential changes cited as important.

\section{NHS/private workload}

Prior to this review, the only available evidence, based on earnings, seemed to suggest that many women dentists in general dental practice work no more than the equivalent of two days per week for the NHS. Of the 3,027 practising GDPs who responded to the survey, $49 \%$ combine NHS and private dentistry, 45\% practise only NHS dentistry whilst $4 \%$ undertake only private dentistry. Of those contemplating a change in balance between NHS and private dentistry (35\% of practising women GDPs), 99\% would shift the balance in favour of private dentistry.

\section{Returning to dentistry}

Of the 529 survey respondents who were not practising dentistry, 233 were either retired, of retirement age or taking early

Table 3 Reasons for survey respondents working part-time by whether currently practising In current post, currently working (\%) In last post, not currently working (\%)

Caring for children

Caring for other relation

65

III-health

Personal choice

Work outside dentistry

Other

(No answer)

Base: number of survey respondents (=100\%)

$\begin{array}{rr}65 & 54 \\ 3 & 6 \\ 4 & 5 \\ 57 & 61 \\ 4 & 6 \\ 13 & 9 \\ 2 & 2 \\ 63 & 330\end{array}$

As survey respondents could choose more than one field the percentages can exceed 100. tinue to subscribe to a dental journal. Only $41 \%$ of the questionnaire survey respondents knew about KITS even though it was introduced in 1988. Only 4\% of survey respondents who had taken a career break had enrolled on the scheme.

Awareness of the Getting Back to Practice (GBTP) course was the same as for KITS (41\%). Uptake was poor with only $4 \%$ of those taking a career break attending a GBTP course.

\section{Practice ownership}

Thirty-two per cent of women general practitioners in the survey (964) owned a dental practice. The benefits perceived by practice owners were wide ranging; at least five out of six practice owners saw benefits in deciding working hours, determining working environment, running the practice how they wanted, determining the work distribution and being able to build their own team.

Practice ownership did not seem to be an overriding ambition of women dentists. Only 27\% of non-practice owners said they would like to own a practice. Those who said 'no' gave a variety of reasons including not being business minded, happy in their current situation and that owning a practice would not fit in with the family and husband's/partner's job. Many mentioned that the paperwork, responsibility and commitment were 'too much hassle' and that they enjoyed their freedom. This included some who had previously been practice owners. It was also made clear that to own a practice and only work part-time was not an attractive financial option. (This is an important point to bear in mind if there are an increase number of women who want to work substantially less than full-time.) Husband and wife ownership of a dental practice is not unusual; in the survey it was seen to occur more markedly in those women over 35 years of age.

\section{Career satisfaction and advice}

Two-thirds of questionnaire survey respondents said their career in dentistry had fulfilled their expectations (Fig. 2), and that they would choose to study dentistry again. A similar proportion had recommended dentistry to other women. Overall, $80 \%$ of those who would choose to be a dentist again said they would decide to practice in the same field.

Only one-quarter of the survey respondents had received career advice as undergraduates. However, there has been a marked improvement over the last four years, reaching $82 \%$ for those who graduated in 2000. The most recent graduates (July 2000) reported receiving useful information about all spheres of practice. 


\section{Community Dental Service}

Sixteen per cent of respondents currently practising worked in the Community Dental Service (CDS). The two most popular reasons for women choosing the CDS as a career were the nature of the work, treating special needs patients and children or that the post was salaried (76\%). Other attractive features recorded in the survey were regular 'office hours' (59\%), the ability to secure part-time work (47\%) and a formal career structure (27\%). (Respondents could tick more than one item.)

\section{Hospital Dental Service and Academic Dentistry}

Among the respondents to the questionnaire survey, $11 \%$ worked in the hospital dental service. Job titles in the hospital dental service covered a wide range including house officer, clinical assistant, associate specialist, specialist registrar and consultant. In the written submissions it was reported that it is not as difficult to develop a career in the hospital services as it had once been due to the introduction of part-time and flexible training opportunities. This has enabled all women to pursue the same goals as male colleagues but evidence from the survey confirms that there are still few women who reach senior positions.

Of the respondents to the questionnaire survey, 5\% held a university appointment. These included professor, consultant, research fellow, tutor and demonstrator.

In written submissions, there were comments that although academic dentistry could offer a rewarding career for women dentists there was little flexibility in university clinical posts and career pathways were based on traditional male working patterns. However, in the focus groups some participants considered it possible to acquire flexibility through organising their work pattern. The training pathways and the necessity for substantial research and postgraduate qualifications to reach the highest posts were considered obstacles when combined with raising a family.

\section{RESULTS - FOCUS GROUPS, PERSONAL SUBMISSIONS AND RESPONSES}

Respondents were also invited to write their own 'free comments' in the questionnaire. These responses, and the findings of the eight focus groups, helped to provide further insights into the statistics compiled from the questionnaires. A total of 64 dentists participated in the eight focus groups. They were selected through contact with local postgraduate tutors, dental practice advisers and clinical community directors. Personal submissions were also received. The report is

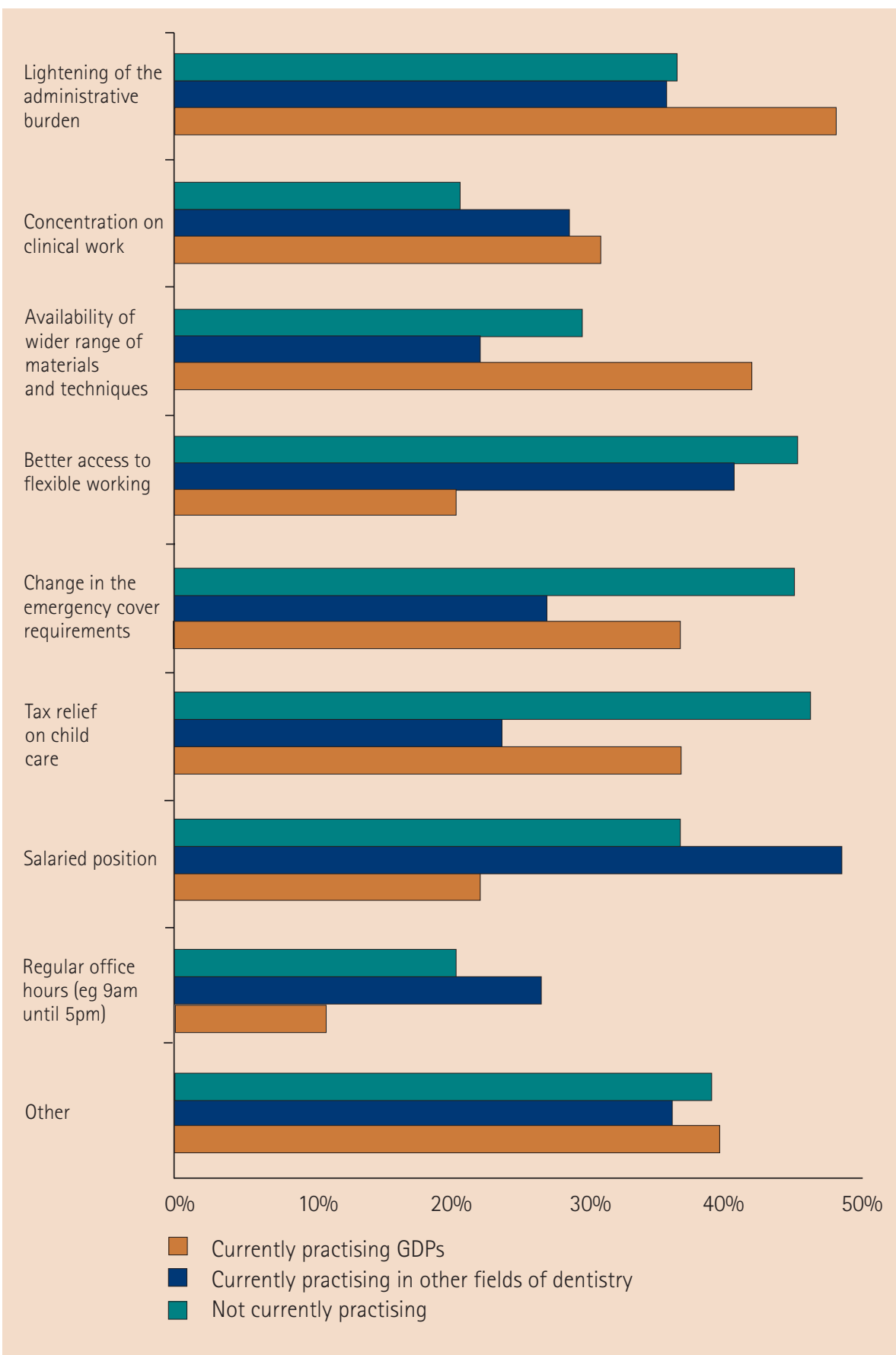

Fig. 1 Potential changes that would attract respondents to work more hours in the GDS.

sprinkled with vignettes, covering all Working patterns major issues.

'I do not want to work more as it would

$(\mathrm{QR}=$ questionnaire response, $\mathrm{FG}=$ focus mean increasing my hours in school holigroup, PS = personal submission) days, which is not fair on the children.' (QR)

Table 4 Reasons given by survey respondents for not currently practising Reason 


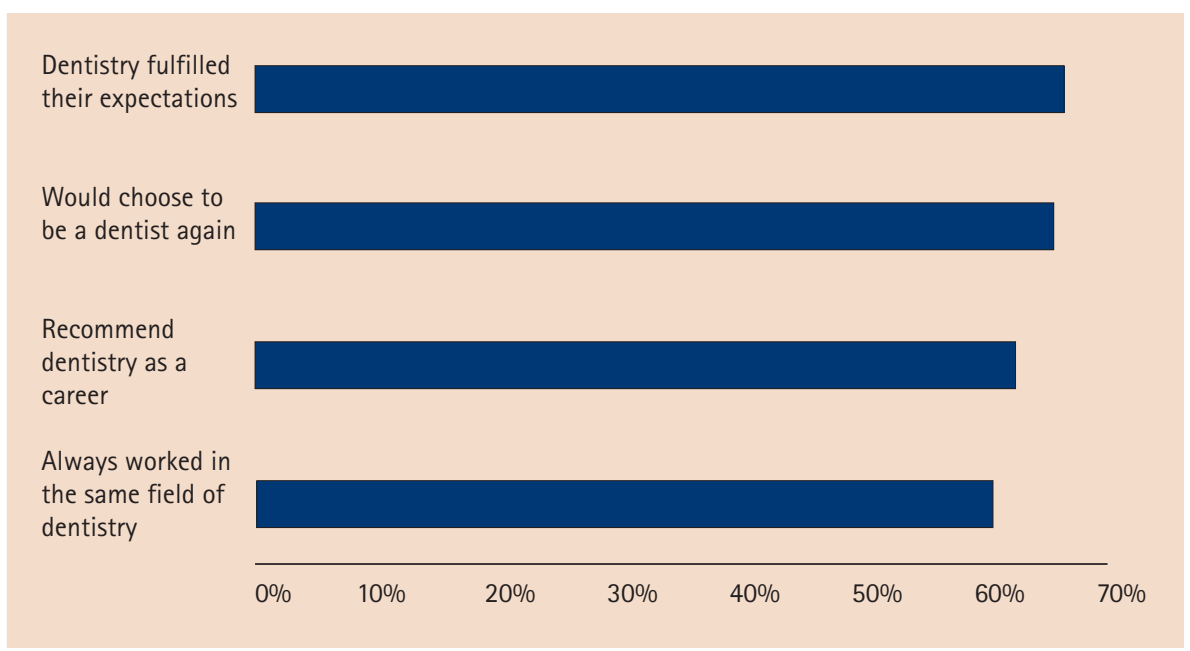

Fig. 2 Survey respondents' views of a dental career.

'I have worked part-time since 24 months' post-qualification. I found full-time general practice very stressful, unpleasant and overtly time consuming.' (QR)

'I found it very difficult to get a parttime post and it was impossible to get a part time associate....' (QR)

\section{NHS/private workload}

'I would only be attracted back to NHS dentistry by no longer having to work conveyor belt dentistry. (QR)

'It's ridiculous. Sometimes there is no way you can make your hourly rate in the NHS.... It conditions how you book a patient, you have to make $£ 1$ a minute.' (FG)

'NHS dentistry is no longer providing the best treatment for patients, because the system will not pay for the best, and why should my patients receive second best.' (QR)

'I have just this week started to convert my NHS practice to Denplan and private dentistry because I am just worn out emotionally and financially after 22 years working more or less full time in the NHS. (PS)

\section{Emergency Cover Arrangements}

'If my husband is away and I'm on call I take the children out of bed.' (FG)

'It's awkward. Sometimes I take the children with me.' (FG)

'Many women are anxious about reopening practices at night or at weekend without other staff available to provide security....There are concerns about parking in some areas which, although safe by day, are not at night.' (PS)

\section{Personal Dental Services (or salaried employment)}

'These should appeal to women wanting to return to general dental practice. There is no financial commitment, a slower pace of work, no on-call or worries about practice management.' (PS)
'We are about to join third-wave PDS, with the whole of our CDS plus an access centre, and I have been absolutely amazed by the number of applicants.' (PS)

The provision of more salaried posts within dentistry would probably have an attraction for many women (as well as men). (PS)

\section{Returning to dentistry}

'Lack of confidence, lack of respect, updated knowledge, family conflicts.' (PS)

'It's difficult to reconcile doing dentistry and leaving children.' (FG)

'It's extremely difficult to take a career break and return on the same footing.' (PS)

\section{Keeping in Touch Scheme}

'Every encouragement should be given to those who wish to return, for whatever reason.' (PS)

'I've never heard of KITS. It could have changed the way I did my retraining.' (FG)

\section{Getting Back to Practice}

'Refresher training needs to be local and adaptable to enable those with families to attend. This is relevant for those who do not live in centres where the courses are traditionally run...' (PS)

'I found GBTP very useful - would have liked more hands-on.' (FG)

\section{Vocational Training}

'I qualified pre-VT. I'm penalised because I specialised for almost 10 years. Now I want to practice in the NHS (rather than hospital) but I don't have a VT number.' (FG)

'I know people who might go back if the requirements to get a VT number weren't so difficult.' (FG)

\section{Practice ownership}

'Having your own practice is nice because you can have your own choices, choose your own staff, influence the situations.' (FG)

'Having surgery and house in one means I can take care of children easier as they are next door.' (QR)

'I can only be a practice owner because my husband and I share the burden.' (QR)

'Male colleagues usually have a wife to support them. In the next life I am going to have a wife to support me!' (PS)

'If not operated full time and with a realistic staffing level the whole thing falls apart unless you are always present.' (PS)

'It is unlikely that I would have a practice if I was not married to a dentist.' (QR)

\section{Career satisfaction and advice}

'I still get great satisfaction in helping a patient who comes in hating their teeth, finish a course of treatment and actually ends up liking the result. I realised this is what I missed the most while being away from dentistry.' (PS)

'Dentistry is an extremely stressful occupation.' (QR)

'It is possible to get a job in most parts of the country, so if you wish to follow a partner for work you can probably get a job without too much difficulty.' (PS)

'I would not hesitate to recommend it to a teenager asking if I would pursue the same career again.' (PS)

'I wouldn't put my children off but I wouldn't encourage them.' (FG)

\section{Community Dental Service}

'I stayed in the CDS while I was having children until we decided our family was complete, because it offers a steady income with maternity benefits, sick pay and postgraduate training without additional cost.' $(\mathrm{QR})$

'That's why I am in a salaried CDS post you don't need a VT number.' (FG)

\section{Hospital dental services and Academic dentistry}

'I am considering a move into hospital dentistry and colleagues have warned me that it is more difficult for a female to firstly get access into hospital posts and then further their hospital career.' (PS)

'Women who wish to proceed to higher hospital and university appointments are definitely at a disadvantage. It is only those who are highly motivated and who can give considerable time commitment to specialist training who are likely to succeed. Inevitably, if there has been either a part- or full-time career break this is a disadvantage when competing with male colleagues in job applications.' (PS)

'I work a 60-hour week. I've got the flexibility that I don't have to do fixed hours so I come home, see my children, and then work until midnight. I have a full-time person living in the house because I can't do it [academic dentistry] otherwise.' (FG) 


\section{Additional relevant issues}

'Many Asian women are now in the profession and many leave work on marriage/ children due to cultural obligations.' (PS)

'For cultural reasons, many Asian women choose to continue to live with their parents when they start working, and this can create difficulties for them during the VT year.' (FG)

'My main grouse is that, because I work a short week, I do not qualify for seniority payments as a GDP.' (QR)

'The stress of NHS practice nearly destroyed my love of the job. (PS)

'The LDC has not made much effort to attract women. It is not representative of the female dental population. It is making decisions that will affect women dentists but females are not involved in the decisions.' (FG)

'Need: practitioners not discriminating against dentists wishing to work only parttime.' (PS)

\section{RESULTS - SUBMISSIONS FROM ORGANISATIONS}

Letters were sent to 54 leading organisations asking for responses to seven questions covering the areas outlined in the Introduction. Because of the coverage given to the questions through press releases to dental publications, a total of 119 written responses were received. The responses are summarised in Annex A of the report. The three areas where most suggestions were made were:

- Working practices: (47 suggestions, with the emphasis on flexible working patterns).

- Financial: (34 suggestions, of which 23 concerned improving/reforming GDS remuneration).

- Childcare: (14 suggestions)

\section{DISCUSSION}

This report reviewed the contribution of women dentists to the workforce. Determining attitudes of people via questionnaires and focus groups is not straightforward, particularly when one of the aims of the study was to summarise the views of women not currently practising dentistry. The overall response rate for completing questionnaires was 55\%, which is very creditable. The results give the most authoritative overview of women dentists' current working patterns and their aspirations for the future. They provide information on which the seven areas referred to in the Introduction can be discussed.

Experience of women dentists in the GDS The trend for an increasing proportion of women to work in general dental practice has continued, from 54\% in 1975 , to $63 \%$ in 1985 , up to $76 \%$ in 2000 . Over half (58\%) work as associates and 5\% as assistants. Only 32\% of women general dental practitioners are practice owners, frequently coowning a practice with their husband/ partner.

\section{Reasons behind the decision to work part- time}

Nearly half the women responding to the survey were working part-time (47\%). This study showed that $92 \%$ of those who tried were successful in obtaining part-time work. Two-thirds of women wanted to work part-time because of caring for children. A small proportion chose to work part-time because of caring for 'other relations' (3\%), personal ill health (4\%) or work outside dentistry (4\%).

\section{Attractiveness of the CDS to women dentists}

Comparison with earlier studies shows a continuing trend of a decrease in the proportion of practising women dentists in the CDS, from $52 \%$ in 1975 , to $38 \%$ in 1985 , to $16 \%$ in 2000. Working in the CDS is attractive to women because it is salaried and involves the treatment of special needs patients and children.

A possible attraction for the CDS lies in the concerns women dentists have raised about working in the GDS. The requirement for dentists in the GDS to provide 24-hour emergency cover has clearly caused women anxiety. The difficulties associated with arranging childcare when called out to re-open a dental practice at night or at the weekend were coupled with concerns for personal safety.

\section{Opportunities provided by the PDS}

The survey showed that only $2 \%$ of women worked in the PDS in 2000. It could be attractive to women as it provides salaried employment and relieves dentists of the administrative and management side of 'running a business', which was consistently referred to as a burden by those responding to the survey.

\section{Challenges women face in returning to work after a career break}

Very few women dentists (8\%) would like to work full time if they returned to practise after a career break. Most (44\%) wanted part-time regular hours or part-time, term time only (37\%). Loss of confidence was repeatedly mentioned as a major obstacle and an anticipated difficulty to overcome concerning returning to work. The issues involved in those women who had actually returned to work after a career break were leaving young children, arranging childcare and loss of confidence.

\section{Usefulness of KITS and GBTP courses}

Only 4\% of the respondents had enrolled on the Keeping in Touch Scheme. From the survey there was a clear message for the scheme to be realistically funded. It is similarly disappointing that Getting Back to Practice courses were only used by $4 \%$ of dentists. Three-quarters of the dentists in the survey who had taken a career break made their own arrangements to keep up to date, using a variety of methods, including reading professional journals, postgraduate meetings, hands-on course, computer assisted learning and distance learning packages.

\section{Other relevant issues}

Overall, 65\% of women dentists said they would choose dentistry again and indicated that their professional career had lived up to their expectations. A similar proportion also made it clear that they would recommend dentistry as a career to other women.

The experience of women undertaking vocational training was raised in focus groups. One problem was the potential difficulty of obtaining a VT placement away from home; the other was the lack of a vocational training number for women who wished to return to NHS general dental practice after a career break and who had ceased to practise before 1997.

Stress was repeatedly mentioned by women dentists in this study. Women said that the pressures of working under the current NHS system of remuneration deterred them from increasing their NHS commitment. Ninety-nine per cent of the practising GDPs contemplating a change in balance between NHS and private dentistry would shift in favour of private dentistry.

\section{CDO'S RECOMMENDATIONS}

Dame Margaret Seward made 24 recommendations, which centred around five issues: working patterns; returning to dentistry; practise ownership; career satisfaction and advice; additional relevant issues.

\section{Working patterns}

1. Review the current arrangements for NHS dentistry (including the current GDS remuneration system) to take account of criticisms made in this review of the obstacles to adopting a more preventive approach and the intensity of the dentist's working day.

2. Investigate ways of increasing the availability of positions with 'employed status' and flexible and part-time working patterns.

3. Consider whether the new out-of-hours arrangements can be designed to meet the concerns of women dentists in rela- 
tion to the current emergency dental cover arrangements.

4. Consider the establishment of 'Bridge the Gap' registers so that dentists can be available at short notice to cover, for example, the illness of children.

\section{Returning to dentistry}

1. Extend eligibility of the Keeping in Touch Scheme (KITS) to dentists who take a break for less than 12 months and review the adequacy of the annual KITS allowance.

2. Develop further the central co-ordination of Getting Back to Practice (GBTP) courses to improve access and availability throughout the country.

3. Build upon the recent experience of the GBTP residential and fully-funded pilot course for all members of the dental team.

4. Improve access to 'hands-on' courses, which provide practical experience in general, and for those on career breaks and for returners in particular.

5. Appoint a Retaining and Returning Adviser in each postgraduate dental deanery who would help dentists to overcome many of the obstacles and assist in finding suitable work. An evaluation of their effectiveness should be planned in advance using an agreed protocol to achieve consistency across deaneries.

6. Pilot a General Dental Practice Retainer Scheme based on the medical model.

7. Pilot a Returners' Vocational Experience Scheme and other career break initiatives.

\section{Practice ownership}

1. Encourage dentists to undertake highquality practice management courses as part of continuing professional development, including through distance learning.

2. Investigate the feasibility of a health authority offering a part-time fixed price PDS contract to a new practice owner to help during the first few years of establishing a practice.

3. Explore the feasibility and benefits of a capital funding system for dentists along the lines of general medical practice initiatives, the General Practice Finance Scheme and NHS LIFT.

4. Explore a system to provide practice management support which will free practice owners to concentrate on clinical work.

\section{Career satisfaction and advice}

1. Continue to improve advice on careers and managing career breaks for undergraduate dental students.

2. Develop a career planning/management breaks handbook for distribution to all NHS dental practices to ensure a continuum of careers advice is readily available for practitioners.

3. Participate in NHS Careers initiative and publicise web site of 'career planning contacts' for dentists.

4. Explore the introduction of incentives to encourage dentists to take additional qualifications.

5. Identify a 'careers mentor' in each deanery who could be a dental tutor or the Retaining and Returning Adviser, and include the name of the contact in the deanery handbook and web sites.

\section{Additional relevant issues}

1. Take into account the cultural diversity within the dental profession and in relation to workforce planning.

2. Encourage women to participate fully in professional activities, including seeking leadership roles and membership of negotiating teams.

3. Investigate how dentists can be included in NHS childcare initiatives. This could also apply to those undertaking postgraduate courses.

4. Explore whether eligibility for the Continuing Professional Development Allowance could be extended to assis- tants in general practice, particularly in view of the mandatory GDC requirements from January 2002.

\section{CONCLUDING COMMENTS}

Rather than summarising each of the nine chapters in this report, I have presented the results in two sections, quantitative data from postal questionnaires and qualitative data concerning the attitudes and opinions of women dentists, and used these results to reflect on the seven major areas of interest identified in the Introduction. The qualitative data is of particular interest because it was collected in three different ways - free comments in the questionnaire, personal submissions and structural focus groups. The quotations in the report come together to provide the best snapshot available of the views of women dentists in England.

The results of this study are important for women in dentistry, but in my view they are very relevant to men too. Career patterns and employment aspirations are changing; issues such as career breaks, part-time working, financial pressures, stress, continuing professional development, career satisfaction, are not gender specific. This point is acknowledged in the report.

This review of the contribution of women dentists to the workforce builds on the work Dr Seward started twenty-five years ago and provides the most up to date information on which better opportunities for women dentists can be considered in the future.

1 Seward M. Better Opportunities for Women Dentists A Review of the Contribution of Women Dentists to the Workforce. Department of Health, London, 2001.

The tables and figures in this article have been extracted from the report in the above reference. These are Crown copyright material and are reproduced with the permission of the Controller of HMSO and the Queen's Printer for Scotland. 